University of Wollongong

Research Online

Australian Institute for Innovative Materials -

Papers

Australian Institute for Innovative Materials

$1-1-2007$

\title{
Elliptical hole pockets in the Fermi surfaces of unhydrated and hydrated sodium cobalt oxides
}

J Laverock

University of Bristol

S B. Dugdale

University of Bristol

J A. Duffy

University of Warwick

$\mathrm{J}$ Wooldridge

University of Warwick

G Balakrishnan

University of Warwick

See next page for additional authors

Follow this and additional works at: https://ro.uow.edu.au/aiimpapers

Part of the Engineering Commons, and the Physical Sciences and Mathematics Commons

Research Online is the open access institutional repository for the University of Wollongong. For further information contact the UOW Library: research-pubs@uow.edu.au 


\title{
Elliptical hole pockets in the Fermi surfaces of unhydrated and hydrated sodium cobalt oxides
}

\author{
Abstract \\ The surprise discovery of superconductivity below $5 \mathrm{~K}$ in sodium cobalt oxides when hydrated with water \\ has caught the attention of experimentalists and theorists alike. Most explanations for its occurence have \\ focused heavily on the properties of some small elliptically shaped pockets predicted to be the \\ electronically dominant Fermi surface sheet, but direct attempts to look for them have instead cast \\ serious doubts over their existence. Here we present evidence that these pockets do indeed exist, based \\ on bulk measurements of the electron momentum distribution in unhydrated and hydrated sodium cobalt \\ oxides using the technique of $\mathrm{x}$-ray Compton scattering.

\section{Keywords} \\ sodium, elliptical, hole, pockets, surfaces, cobalt, unhydrated, oxides, hydrated, fermi \\ Disciplines \\ Engineering | Physical Sciences and Mathematics

\section{Publication Details} \\ Laverock, J., Dugdale, S. B., Duffy, J. A., Wooldridge, J., Balakrishnan, G., Lees, M. R., Zheng, G., Chen, D., \\ Lin, C. T. \& Sakurai, Y. (2007). Elliptical hole pockets in the Fermi surfaces of unhydrated and hydrated \\ sodium cobalt oxides. Physical Review B: Condensed Matter and Materials Physics, 76 (5), \\ 052509-1-052509-4.
}

\section{Authors}

J Laverock, S B. Dugdale, J A. Duffy, J Wooldridge, G Balakrishnan, M R. Lees, G.-q Zheng, Dapeng Chen, C T. Lin, and Y Sakurai 


\title{
Elliptical hole pockets in the Fermi surfaces of unhydrated and hydrated sodium cobalt oxides
}

\author{
J. Laverock and S. B. Dugdale \\ H. H. Wills Physics Laboratory, University of Bristol, Tyndall Avenue, Bristol BS8 1TL, United Kingdom \\ J. A. Duffy, J. Wooldridge, G. Balakrishnan, and M. R. Lees \\ Department of Physics, University of Warwick, Coventry CV4 7AL, United Kingdom \\ G.-q. Zheng \\ Department of Physics, Okayama University, Okayama 700-8530, Japan \\ D. Chen and C. T. Lin \\ ISEM, University of Wollongong, Wollongong, NSW 2522, Australia
}

\author{
A. Andrejczuk, M. Itou, and Y. Sakurai \\ Japan Synchrotron Radiation Research Institute, SPring-8, 1-1-1 Kouto, Mikazuki, Sayo, Hyogo 679-5198, Japan
}

(Received 1 August 2007; published 22 August 2007)

\begin{abstract}
The surprise discovery of superconductivity below $5 \mathrm{~K}$ in sodium cobalt oxides when hydrated with water has caught the attention of experimentalists and theorists alike. Most explanations for its occurence have focused heavily on the properties of some small elliptically shaped pockets predicted to be the electronically dominant Fermi surface sheet, but direct attempts to look for them have instead cast serious doubts over their existence. Here we present evidence that these pockets do indeed exist, based on bulk measurements of the electron momentum distribution in unhydrated and hydrated sodium cobalt oxides using the technique of $\mathrm{x}$-ray Compton scattering.
\end{abstract}

DOI: 10.1103/PhysRevB.76.052509

PACS number(s): 71.18.+y, 71.27.+a, 74.70.-b

Hydrated sodium cobalt oxides $\left(\mathrm{Na}_{x} \mathrm{CoO}_{2} \cdot 1.3 \mathrm{H}_{2} \mathrm{O}\right)$, for a certain range of $\mathrm{Na}$ concentrations, exhibit superconductivity at a temperature of $5 \mathrm{~K} .{ }^{1}$ Although many analogies have been drawn with the high- $T_{c}$ cuprates (for instance, as being possibly the only other example of a Mott insulator becoming superconducting under doping ${ }^{1,2}$ ), the sodium cobaltate system exhibits its own unique set of anomalous behaviour such as unusually high thermopower ${ }^{3}$ and $T$-linear resistivity $^{4}$ ), distinctly indicative of strongly correlated electron behavior. In a conventional superconductor, electrons at the Fermi surface form Cooper pairs under an attractive interaction mediated by lattice vibrations. The manner in which electrons form these pairs can be strongly influenced by the shape of the Fermi surface. Questions regarding the origin of the pairing interaction and the nature of the superconductivity in the cobaltates has stimulated significant theoretical speculation, most of which has focused heavily on the properties of some small elliptically shaped pockets predicted to be the electronically dominant Fermi surface sheet ${ }^{5-8}$ but the outcome of direct attempts to look for them has instead cast serious doubt over their existence. ${ }^{10-14}$ Here we present evidence that these pockets do indeed exist, based on bulk measurements of the electron momentum distribution in unhydrated and hydrated sodium cobalt oxides using the technique of $\mathrm{x}$-ray Compton scattering.

The structure of $\mathrm{Na}_{x} \mathrm{CoO}_{2}$ comprises hexagonal planes of electronically active edge-sharing $\mathrm{CoO}_{6}$ octahedra. ${ }^{16}$ These planes are separated by insulating layers of $\mathrm{Na}$, and, in the hydrated samples, water, that serve as spacers (resulting in electronic two dimensionality) and charge reservoirs. The intercalation of water, at a concentration of $y \sim 1.3$, has a dramatic effect on properties of the compound. It is accompa- nied by a near doubling of the $c$-axis lattice parameter and, for $x \sim 0.3$, the onset of superconductivity. Calculations of electronic structure based on the local-density approximation (LDA) predict, for concentrations $x \leqslant \sim 0.6$ in $\mathrm{Na}_{x} \mathrm{CoO}_{2}$, a Fermi surface composed of two hole sheets originating from Co $d$ orbitals. ${ }^{17-19}$ The larger sheet, of $a_{1 g}$ character, is centered at the $\Gamma$ point (zone center) whereas the other sheet, of $e_{g}^{\prime}$ character, comprises six smaller elliptical cylinders located between $\Gamma$ and $K$ (for $x \geqslant 0.7$, this band becomes completely filled and does not contribute to the Fermi surface). These $e_{g}^{\prime}$ pockets may be of considerable importance, since many of the models for superconductivity in $\mathrm{Na}_{x} \mathrm{CoO}_{2} \cdot y \mathrm{H}_{2} \mathrm{O}$ are predicated upon their existence. In particular, an analysis of permitted order-parameter symmetries has indicated that an unconventional $f$ wave $\left[x\left(x^{2}-3 y^{2}\right) \hat{\mathbf{z}}\right]$ would be the most probable if the pockets exist. ${ }^{7}$ With $70 \%$ of the density-ofstates at the Fermi level, these pockets are central to several models of spin-fluctuation-mediated superconductivity, developing either via imperfect nesting between the Fermi surface pockets, ${ }^{5}$ or as a consequence of these disconnected pieces of Fermi surface. ${ }^{6,8}$

A series of angle-resolved photoemission spectroscopy (ARPES) results, the consensus of which report observation of the $a_{1 g}$ sheet at $\Gamma$ but crucially not the $e_{g}^{\prime}$ pockets, has fuelled the controversy surrounding the elliptical pockets. Indeed, they observe the $e_{g}^{\prime}$ band as lying completely below the Fermi level. ${ }^{11-14}$ However, it is well known that surface effects and matrix elements can have a strong influence on photoemission results, although there are recent reports of measurements being made with an electron escape depth of $200 \AA .{ }^{15}$ Here we directly tackle this controversy by presenting a Compton scattering study both of the Fermi surfaces of 
a representative set of compositions of the unhydrated parent compound $\mathrm{Na}_{x} \mathrm{CoO}_{2}(x=0.38,0.51$, and 0.74$)$ and a hydrated (actually deuterated) sample at a superconducting composition $\mathrm{Na}_{0.35} \mathrm{CoO}_{2} \cdot 1.3 \mathrm{D}_{2} \mathrm{O}$. For these measurements, single crystals of $x \sim 0.75$ were grown in Warwick using the floating-zone technique. Samples of lower sodium concentration were then obtained by a chemical deintercalation method, immersing the crystals in solutions of $\mathrm{Br}_{2}$ and acetonitrile. The lattice parameters were obtained by $\mathrm{x}$-ray diffraction; the relationship between $\mathrm{Na}$ doping and the crystal structure is well characterized by powder neutron diffraction measurements and ICP-AES techniques ${ }^{20}$ and so the sodium concentrations for the three crystals used in this study were determined as 0.74(1), 0.51(1), and 0.38(1). The macroscopic properties (magnetic susceptibility, heat capacity, and transport measurements ${ }^{21}$ ) are identical to those previously reported for similar compositions. ${ }^{22}$ The superconducting sample was produced by the chemical intercalation of deuterium oxide $\left(\mathrm{D}_{2} \mathrm{O}\right.$ was used rather than $\mathrm{H}_{2} \mathrm{O}$ to allow future neutron experiments on the same sample) by submersion in liquid $\mathrm{D}_{2} \mathrm{O}$ for three months at $5{ }^{\circ} \mathrm{C} .{ }^{23}$ Subsequent to the Compton experiment, a measurement of its magnetisation showed that it was superconducting at a temperature of $3.5 \mathrm{~K}$.

A Compton profile represents a double integral (onedimensional projection) of the full three-dimensional electron momentum density. For each composition, five Compton profiles equally spaced between $\Gamma-M$ and $\Gamma-K$ were measured on the high-resolution Compton spectrometer of beamline BL08W at the SPring-8 synchrotron. The unhydrated measurements were made at room temperature, while those on the hydrated sample were at $11 \mathrm{~K}$. The hydrated sample was transferred from a $\mathrm{D}_{2} \mathrm{O}$ bath directly onto a cryostat precooled to $\sim 250 \mathrm{~K}$ under a helium atmosphere in order to preserve the hydration. On removal at the end of the measurement, the sample was observed to be still in its hydrated state. The spectrometer consists of a Cauchois-type crystal analyzer and a position-sensitive detector, with a resolution FWHM at the Compton peak of 0.115 a.u. (1 a.u. of momentum $\left.=1.99 \times 10^{-24} \mathrm{~kg} \mathrm{~m} \mathrm{~s}^{-1}\right) .{ }^{24,25}$ For each Compton profile, $\sim 600000$ counts in the peak data channel were accumulated, and each Compton profile was corrected for possible multiple-scattering contributions. A twodimensional momentum density, representing a projection down the $c$ axis of the full three-dimensional density, was reconstructed from each set of five profiles using tomographic techniques ${ }^{26}$ and then folded back into the first BZ using the Lock-Crisp-West procedure ${ }^{27,28}$ to obtain the occupation density from which the occupied parts of the BZ could be inferred. The occupation density is shown for each composition in Fig. 1, where black represents the lowest occupancy and white the highest.

Considering first the unhydrated parent compound, the contours associated with the hexagonal $a_{1 g}$ hole sheet can be clearly identified for $x=0.74$, but the hexagonal shape becomes progressively less clear and is significantly distorted in the $x=0.38$ data. We shall argue that this distortion is strong evidence for the presence of the $e_{g}^{\prime}$ pockets.

To assess the size of the hexagonal Fermi surface of the $\mathrm{Na}_{0.74} \mathrm{CoO}_{2}$ compound, a method using the extrema in the

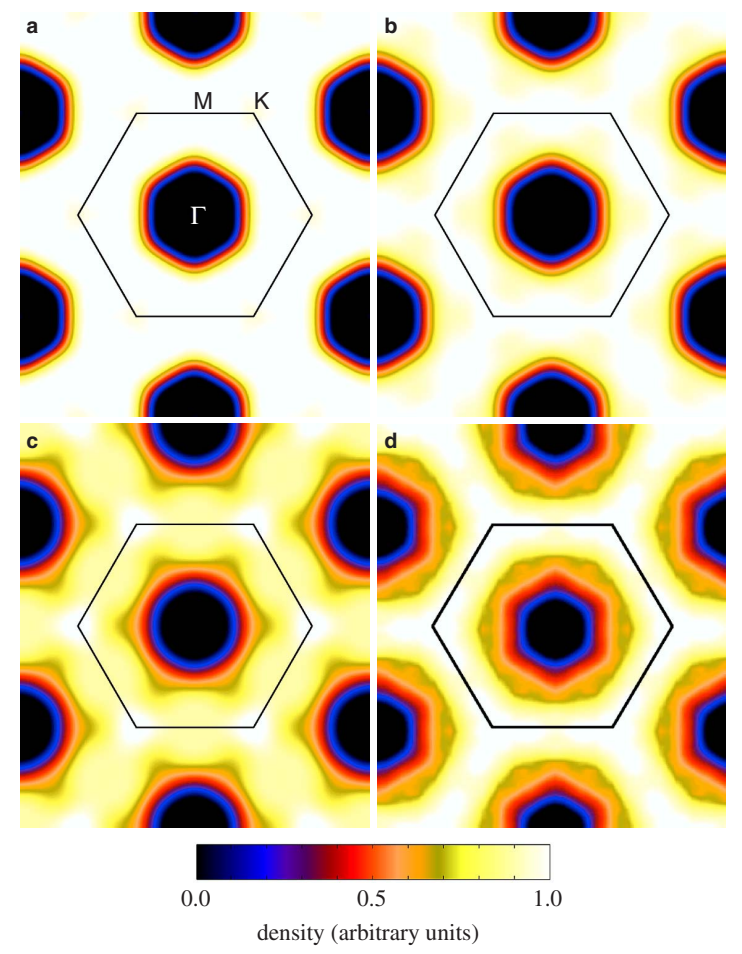

FIG. 1. (Color online) The experimental Fermi surface of $\mathrm{Na}_{x} \mathrm{CoO}_{2}$ for (a) $x=0.74$, (b) 0.51 , and (c) 0.38 , and for $\mathrm{Na}_{0.35} \mathrm{CoO}_{2} \cdot 1.3 \mathrm{D}_{2} \mathrm{O}$ (d) obtained from the reconstruction of five Compton profiles for each composition. The boundary of the first Brillouin zone is indicated.

first derivative of the occupation density was employed (see, for example, Ref. 27). At this composition elliptical pockets are not expected, and so the determination using this method should unambiguously reveal the hexagonal Fermi surface. Figure 2 is the result, and shows a Fermi surface in excellent agreement with LDA calculations.

We can explain the distortion of the hexagonal shape for smaller Na concentrations as being due to the presence of $e_{g}^{\prime}$ elliptical hole pockets close to the central $a_{1 g}$ Fermi surface.

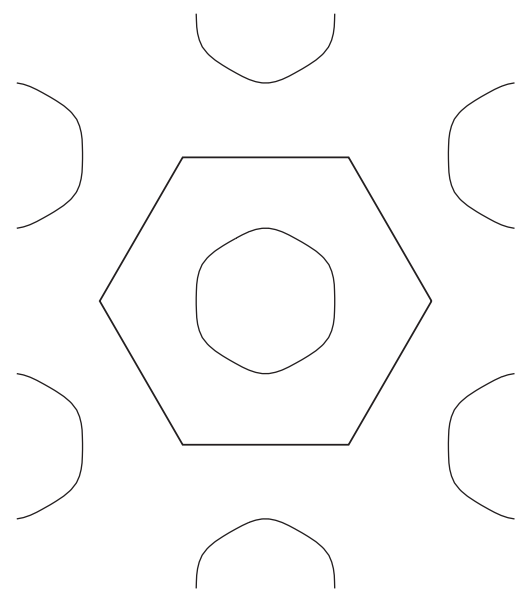

FIG. 2. Fermi surface obtained by plotting the contour at the maximum of the first derivative of the occupation density for $\mathrm{Na}_{0.74} \mathrm{CoO}_{2}$. 


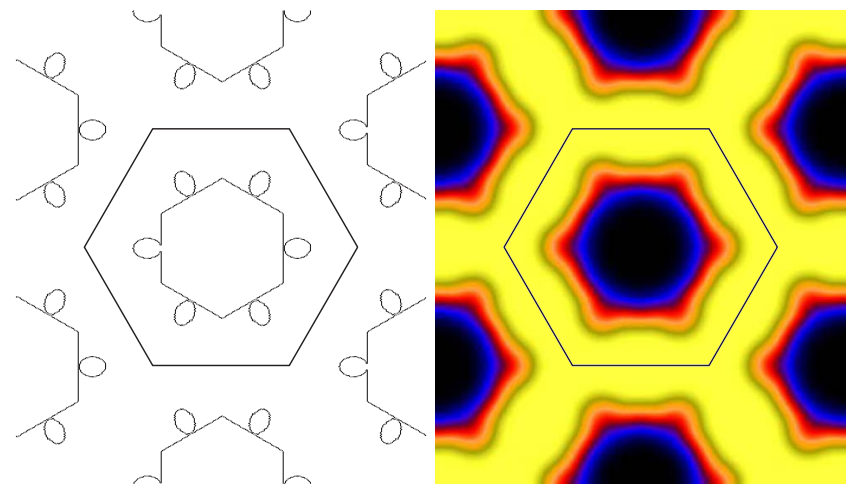

FIG. 3. (Color online) A simulation of a Fermi surface comprising a central hexagonal sheet (representing the $a_{1 g}$ hole sheet) and six $e_{g}^{\prime}$ elliptical hole pockets (left) together with the resulting occupation density convoluted with the experimental resolution (right).

A simple geometric simulation of such a Fermi surface is shown in Fig. 3 together with the resulting occupation density (convoluted with the experimental resolution), illustrating how the presence of small pockets distort the hexagonal appearance of the $a_{1 g}$ Fermi surface. The experimental occupation density for the hydrated $\mathrm{Na}_{0.35} \mathrm{CoO}_{2} \cdot 1.3 \mathrm{D}_{2} \mathrm{O}$ (Fig. 1) is also strongly suggestive of the presence of small hole pockets, which can be discerned close to the central hexagon; it is also worth remarking that the $a_{1 g}$ sheet retains a strong hexagonal shape, whereas electronic structure calculations in the hydrated structure (although without the actual presence of water) predict something more circular. ${ }^{29}$ Our results suggest that the effects of hydration on the Fermi surface are in fact rather modest, and perhaps not as drastic as suggested by Xiao et al. ${ }^{30}$ An estimate of the areas of the hexagonal $a_{1 g}$ sheet and (where appropriate) the six elliptical $e_{g}^{\prime}$ pockets based on a comparison of simulations to the experimental data (with the total area constrained by the appropriate $\mathrm{Na}$ concentration) is presented in Table I. That the pockets consistently appear rather close to the $a_{1 g}$ sheet is also noteworthy.

Recent measurement of Shubnikov-de Haas oscillations in $\mathrm{Na}_{0.3} \mathrm{CoO}_{2}$ indicate the presence of some unidentified Fermi surface pockets occupying approximately 0.6 and $1.4 \%$ of the BZ, ${ }^{31}$ which is consistent with our estimate of the size of each pocket we observe occupying about $0.8 \%$ (Table I). In addition, an examination of phonon softening in this system by Rueff et $a l .{ }^{32}$ is interpreted by those authors as strong evidence for the existence of nested pockets.

The question of why the ARPES experiments have consistently not observed these $e_{g}^{\prime}$ pockets remains. Issues such as surface sensitivity, including possible surface relaxations of $\mathrm{CoO}_{6}$ octahedral contractions that destroy the pockets, $, 9,33$ as well as matrix-element effects or $\mathrm{Na}$ disorder ${ }^{34}$ must be possibilities. However, at least in the superconducting compound, it is very difficult to reconcile the observed behavior
TABLE I. Estimate of areas of the $a_{1 g}$ and $\mathrm{e}_{g}^{\prime}$ Fermi surfaces (in the case of the latter, this is the total area of all six pockets) based on applying the simulation of the Fermi surface as illustrated in Fig. 3 for the compositions studied, as a proportion of the hexagonal first Brillouin zone.

\begin{tabular}{lcc}
\hline \hline Cobaltate & Area $\left(a_{1 g}\right)$ & Area $\left(\mathrm{e}_{g}^{\prime}\right)$ \\
\hline $\mathrm{Na}_{0.38} \mathrm{CoO}_{2}$ & 0.262 & 0.048 \\
$\mathrm{Na}_{0.51} \mathrm{CoO}_{2}$ & 0.218 & 0.027 \\
$\mathrm{Na}_{0.74} \mathrm{CoO}_{2}$ & 0.132 & not seen \\
$\mathrm{Na}_{0.35} \mathrm{CoO}_{2} \cdot 1.3 \mathrm{D}_{2} \mathrm{O}$ & 0.310 & 0.016 \\
\hline \hline
\end{tabular}

of the specific heat, or even understand the presence of superconductivity without the presence of the $e_{g}^{\prime}$ pockets. ${ }^{35}$ Moreover, in an attempt to take into account Coulomb correlations not included in the LDA, calculations ${ }^{18,19}$ based on the LDA $+U$ approach, have suggested that for a sufficiently large Coulomb energy $(U>3 \mathrm{eV})$ the $e_{g}^{\prime}$ band is pulled below the Fermi level, eliminating these smaller Fermi surface pockets for all $\mathrm{Na}$ concentrations. However, other studies have put an upper limit of about $2.3 \mathrm{eV}$ on $U,^{36}$ and when dynamical Coulomb correlations are incorporated the effect is to stabilize the $e_{g}^{\prime}$ pockets. ${ }^{37}$ The theoretical debate rages on, with predictions in support of (Ref. 38) and contrary to (Ref. 39) the existence of these pockets.

In conclusion, we have presented the Fermi surface of several members $(x=0.38,0.51$, and 0.74$)$ of the unhydrated sodium cobalt oxide $\mathrm{Na}_{x} \mathrm{CoO}_{2}$ and of a hydrated composition $\mathrm{Na}_{0.35} \mathrm{CoO}_{2} \cdot 1.3 \mathrm{D}_{2} \mathrm{O}$. Reasonable qualitative agreement is observed between our experimentally determined Fermi surfaces and the LDA predictions, and there is clear evidence for the smaller $e_{g}^{\prime}$ elliptical hole pockets which develop at lower $\mathrm{Na}$ concentrations than $\mathrm{Na}_{0.74} \mathrm{CoO}_{2}$. For $\mathrm{Na}_{0.38} \mathrm{CoO}_{2}$, their presence is clearly indicated in experimental maps of the occupancy within the Brillouin zone. Most importantly, however, the occupancy map for $\mathrm{Na}_{0.35} \mathrm{CoO}_{2} \cdot 1.3 \mathrm{D}_{2} \mathrm{O}$ also shows the presence of small $e_{g}^{\prime}$ elliptical hole pockets. While alternative models that describe the superconductivity arising as a consequence of frustration on the triangular lattice, ${ }^{40,41}$ nesting across the large, hexagonal sheet, ${ }^{42}$ or spin fluctuations enhanced by the dopant dynamics ${ }^{43}$ are not (and cannot be) ruled out, the observation of the pockets lends strong support to theories based on their special nesting properties.

We acknowledge the financial support of the Royal Society (S.B.D.) and the UK EPSRC, and invaluable discussions with Igor Mazin, Michelle Johannes, and Zahid Hasan. This experiment was performed with the approval of the Japan Synchrotron Radiation Research Institute (JASRI, proposal Nos. 2005A0092-ND3a-np and 2005B0182-ND3a-np). This work was partially supported by a Grant-in-Aid for Scientific Research (Grant No. 18340111) from the Ministry of Education, Culture, Sports, Science and Technology, Japan. 
${ }^{1}$ K. Takada, H. Sakurai, E. Takayama-Muromachi, F. Izumi, R. A. Dilanian, and T. Sasaki, Nature (London) 422, 53 (2003).

${ }^{2}$ Q. H. Wang, D. H. Lee, and P. A. Lee, Phys. Rev. B 69, 092504 (2004).

${ }^{3}$ I. Terasaki, Y. Sasago, and K. Uchinokura, Phys. Rev. B 56, R12685 (1997).

${ }^{4}$ Y. Y. Wang, N. S. Rogado, R. J. Cava, and N. P. Ong, Nature (London) 423, 425 (2003).

${ }^{5}$ M. D. Johannes, I. I. Mazin, D. J. Singh, and D. A. Papaconstantopoulos, Phys. Rev. Lett. 93, 097005 (2004).

${ }^{6}$ K. Kuroki, Y. Tanaka, and R. Arita, Phys. Rev. Lett. 93, 077001 (2004).

${ }^{7}$ I. I. Mazin and M. D. Johannes, Nat. Phys. 1, 91 (2005).

${ }^{8}$ M. Mochizuki, Y. Yanase, and M. Ogata, Phys. Rev. Lett. 94, 147005 (2005).

${ }^{9}$ M. Mochizuki and M. Ogata, J. Phys. Soc. Jpn. 75, 113703 (2006).

${ }^{10}$ M. Z. Hasan, Y. D. Chuang, D. Qian, Y. W. Li, Y. Kong, A. P. Kuprin, A. V. Fedorov, R. Kimmerling, E. Rotenberg, K. Rossnagel, Z. Hussain, H. Koh, N. S. Rogado, M. L. Foo, and R. J. Cava, Phys. Rev. Lett. 92, 246402 (2004).

${ }^{11}$ H. B. Yang, S. C. Wang, A. K. P. Sekharan, H. Matsui, S. Souma, T. Sato, T. Takahashi, T. Takeuchi, J. C. Campuzano, R. Jin, B. C. Sales, D. Mandrus, Z. Wang, and H. Ding, Phys. Rev. Lett. 92, 246403 (2004).

${ }^{12}$ H. B. Yang, Z. H. Pan, A. K. P. Sekharan, T. Sato, S. Souma, T. Takahashi, R. Jin, B. C. Sales, D. Mandrus, A. V. Fedorov, Z. Wang, and H. Ding, Phys. Rev. Lett. 95, 146401 (2005).

${ }^{13}$ D. Qian, L. Wray, D. Hsieh, D. Wu, J. L. Luo, N. L. Wang, A. Kuprin, A. Fedorov, R. J. Cava, L. Viciu, and M. Z. Hasan, Phys. Rev. Lett. 96, 046407 (2006).

${ }^{14}$ D. Qian, D. Hsieh, L. Wray, Y. D. Chuang, A. Fedorov, D. Wu, J. L. Luo, N. L. Wang, M. Viciu, R. J. Cava, and M. Z. Hasan, Phys. Rev. Lett. 96, 216405 (2006).

${ }^{15}$ T. Shimojima, K. Ishizaka, S. Tsuda, T. Kiss, T. Yokoya, A. Chainani, S. Shin, P. Badica, K. Yamada, and K. Togano, Phys. Rev. Lett. 97, 267003 (2006).

${ }^{16}$ L. Viciu, J. W. G. Bos, H. W. Zandbergen, Q. Huang, M. L. Foo, S. Ishiwata, A. P. Ramirez, M. Lee, N. P. Ong, and R. J. Cava, Phys. Rev. B 73, 174104 (2006).

${ }^{17}$ D. J. Singh, Phys. Rev. B 61, 13397 (2000).

${ }^{18}$ P. H. Zhang, W. D. Luo, M. L. Cohen, and S. G. Louie, Phys. Rev. Lett. 93, 236402 (2004).

${ }^{19}$ S. Zhou, M. Gao, H. Ding, P. A. Lee, and Z. Q. Wang, Phys. Rev. Lett. 94, 206401 (2005).
${ }^{20}$ Q. Huang, M. L. Foo, R. A. Pascal, J. W. Lynn, B. H. Toby, T. He, H. W. Zandbergen, and R. J. Cava, Phys. Rev. B 70, 184110 (2004).

${ }^{21}$ J. Wooldridge, D. M. Paul, G. Balakrishnan, and M. R. Lees, J. Phys.: Condens. Matter 17, 707 (2005).

${ }^{22}$ F. C. Chou, J. H. Cho, and Y. S. Lee, Phys. Rev. B 70, 144526 (2004).

${ }^{23}$ F. C. Chou, J. H. Cho, P. A. Lee, E. T. Abel, K. Matan, and Y. S. Lee, Phys. Rev. Lett. 92, 157004 (2004).

${ }^{24}$ N. Hiraoka, M. Itou, T. Ohata, M. Mizumaki, Y. Sakurai, and N. Sakai, J. Synchrotron Radiat. 8, 26 (2001).

${ }^{25}$ Y. Sakurai and M. Itou, J. Phys. Chem. Solids 65, 2061 (2004).

${ }^{26}$ G. Kontrym-Sznajd, Phys. Status Solidi A 117, 227 (1990).

${ }^{27}$ Zs. Major, S. B. Dugdale, R. J. Watts, G. Santi, M. A. Alam, S. M. Hayden, J. A. Duffy, J. W. Taylor, T. Jarlborg, E. Bruno, D. Benea, and H. Ebert, Phys. Rev. Lett. 92, 107003 (2004).

${ }^{28}$ S. B. Dugdale, R. J. Watts, J. Laverock, Zs. Major, M. A. Alam, M. Samsel-Czekała, G. Kontrym-Sznajd, Y. Sakurai, and M. Itou, D. Fort, Phys. Rev. Lett. 96, 046406 (2006).

${ }^{29}$ M. D. Johannes and D. J. Singh, Phys. Rev. B 70, 014507 (2004).

${ }^{30}$ R. J. Xiao, H. X. Yang, and J. Q. Li, Phys. Rev. B 73, 092517 (2006).

${ }^{31}$ L. Balicas, J. G. Analytis, Y. J. Jo, K. Storr, H. Zandbergen, Y. Xin, N. E. Hussey, F. C. Chou, and P. A. Lee, Phys. Rev. Lett. 97, 126401 (2006).

${ }^{32}$ J. P. Rueff, M. Calandra, M. d'Astuto, Ph. Leininger, A. Shukla, A. Bossak, M. Krisch, H. Ishii, Y. Cai, P. Badica, T. Sasaki, K. Yamada, and K. Togano, Phys. Rev. B 74, 020504(R) (2006).

${ }^{33}$ M. Mochizuki, Y. Yanase, and M. Ogata, J. Phys. Soc. Jpn. 74, 1670 (2005).

${ }^{34}$ D. J. Singh and D. Kasinathan, Phys. Rev. Lett. 97, 016404 (2006).

${ }^{35}$ N. Oeschler, R. A. Fisher, N. E. Phillips, J. E. Gordon, M. L. Foo, and R. J. Cava, Chin. J. Phys. (Taipei) 43, 574 (2005).

${ }^{36}$ K. W. Lee and W. E. Pickett, Phys. Rev. B 72, 115110 (2005).

${ }^{37}$ H. Ishida, M. D. Johannes, and A. Liebsch, Phys. Rev. Lett. 94, 196401 (2005).

${ }^{38}$ H. Ishida and A. Liebsch, arXiv:0705.3627 (unpublished).

${ }^{39}$ A. Bourgeois, A. A. Aligia, T. Kroll, and M. D. Nunez-Regueiro, Phys. Rev. B 75, 174518 (2007).

${ }^{40}$ B. Kumar and B. S. Shastry, Phys. Rev. B 68, 104508 (2003).

${ }^{41}$ M. Ogata, J. Phys. Soc. Jpn. 72, 1839 (2003).

${ }^{42}$ A. Tanaka and X. Hu, Phys. Rev. Lett. 91, 257006 (2003).

${ }^{43}$ G. Baskaran, Phys. Rev. Lett. 91, 097003 (2003). 